\title{
Enhanced immunization via dissolving microneedle array-based delivery system incorporating subunit vaccine and saponin adjuvant
}

This article was published in the following Dove Press journal:

International Journal of Nanomedicine

4 July 2017

Number of times this article has been viewed

\author{
Ji-Hui Zhaol,* \\ Qi-Bo Zhangl,* \\ Bao Liu² \\ Xiang-Hua Piao' \\ Yu-Lu Yan' \\ Xiao-Ge Hu' \\ Kuan Zhou' \\ Yong-Tai Zhang' \\ Nian-Ping Feng' \\ 'School of Pharmacy, Shanghai \\ University of Traditional Chinese \\ Medicine, Shanghai, People's Republic \\ of China; ${ }^{2}$ Anethesiology Department, \\ Augusta University, Augusta, GA, USA \\ *These authors contributed equally \\ to this work
}

Correspondence: Nian-Ping Feng School of Pharmacy, Shanghai University of Traditional Chinese Medicine, Shanghai 20I203, People's Republic of China Email npfeng@hotmail.com
Purpose: To enhance the immunogenicity of the model subunit vaccine, ovalbumin (OVA) was combined with platycodin (PD), a saponin adjuvant. To reduce the toxicity of PD, OVA, and adjuvant were loaded together into liposomes before being incorporated into a dissolving microneedle array.

Methods: OVA- and PD-loaded liposomes (OVA-PD-Lipos) were prepared using the film dispersion method. Their uptake behavior, toxicity to mouse bone marrow dendritic cells (BMDCs), and hemolytic activity to rabbit red blood cells (RBCs) were evaluated. The OVA-PD-Lipos were incorporated into a dissolving microneedle array. The chemical stability of OVA and the physical stability of OVA-PD-Lipos in microneedle arrays were investigated. The immune response of Institute of Cancer Research mice and potential skin irritation reaction of rabbits to OVA-PD-Lipos-MNs were evaluated.

Results: The uptake of OVA by mouse BMDCs was greatly enhanced when OVA was prepared as OVA-PD-Lipos, and in this form, the toxicity of PD was dramatically reduced. OVA was chemically stable as OVA-PD-Lipos, when OVA-PD-Lipos was incorporated into a dissolving microneedle array. Institute of Cancer Research mice treated with OVA-PDLipos-MNs showed a significantly enhanced immune response. PD combined with OVA elicited a balanced Th1 and Th2 humoral immune response in mice, with minimal irritation in rabbit skin.

Conclusion: The dissolving microneedle array-based system is a promising delivery vehicle for subunit vaccine and its adjuvant

Keywords: subunit vaccine, saponin adjuvant, liposomes, dissolving microneedle array, intradermal vaccination

\section{Introduction}

Subunit vaccines provide excellent safety but poor immunogenicity. The primary strategies for enhancing immunogenicity are to combine antigens with adjuvants and to incorporate the mixture into nanoscale delivery systems to facilitate uptake and presentation by dendritic cells (DCs). However, adjuvants can introduce health risks. For example, saponins are a chemically heterogeneous group of steroid and triterpenoid glycosides present in a wide range of plant species, characterized as potent adjuvants enhancing both Th1 (humoral) and Th2 (cellular) responses. Quillaia saponin and its derivative Iscomatrix ${ }^{\mathrm{TM}}$ are widely used in animal vaccines, but are not considered suitable for human use because of their toxicity. ${ }^{1}$ Recently, adjuvants based on saponins from traditional Chinese medicinal herbs have been described. ${ }^{2}$ One promising example is platycodin (PD), it exhibits potent adjuvant activity with less toxicity than 
Quillaia saponin. ${ }^{3}$ It is a potential adjuvant for human use, but its toxicity still exceeds acceptable levels.

DCs play a vital role in the process of immune response elicitation mediated by vaccines. ${ }^{4,5}$ Although subcutaneous and intramuscular routes are typically used for vaccination, skin harbors a widespread network of DCs, which contains a well-developed immune system with good connection to regional lymphatic tissues, making it an attractive site for efficient immunization. ${ }^{6}$ Because intradermal vaccination can elicit more potent immune responses than subcutaneous or intramuscular vaccination, its use has spread rapidly. ${ }^{6,7}$ Although conventional intradermal vaccination involves injection with a syringe needle, it is possible to incorporate vaccines into dissolving microneedles. This approach to intradermal vaccination offers better patient compliance, reliability, and simplicity. ${ }^{8-11}$

In this study, the model antigen ovalbumin (OVA) and the adjuvant PD were loaded into liposomes to facilitate uptake of the vaccine by DCs and to reduce the toxicity of the adjuvant. The uptake behavior and toxicity of the OVA- and PD-loaded liposomes (OVA-PD-Lipos) were evaluated using cultured mouse bone marrow dendritic cells (BMDCs), and their hemolytic activity was evaluated in rabbit red blood cells (RBCs). To enhance vaccination efficiency, OVA-PD-Lipos were incorporated into dissolving microneedles. The immunization efficiency of the OVA-PDLipos-incorporated microneedles (OVA-PD-Lipos-MNs) was investigated in mice, and their ability to provoke skin irritation was evaluated using rabbits.

\section{Materials and methods Reagents}

OVA (grade VII), granulocyte macrophage-colony stimulating factor (GM-CSF) from mouse, 2-mercaptoethanol, cholesterol, Sephadex $^{\circledR}$ G-50, and octadecylamine were purchased from Sigma-Aldrich (St Louis, MO, USA). PD (98\%) was obtained from Meilunbio (Dalian, China). Hydrogenated egg phosphatidylcholine (HEPC) was obtained from AVT (Shanghai, China). Polyvinylpyrrolidone-K17 (PVP-K17) and PVP-K30 were provided by BOAI NKY Pharmaceuticals Ltd. (Tianjin, China).

\section{Preparation and characterization of OVA-PD-Lipos}

OVA- and PD-loaded liposomes were prepared using the film dispersion method, and their particle size was reduced by filtration through polycarbonate membranes. ${ }^{12}$ Briefly, HEPC (13.0 mg), cholesterol (7.0 mg), and octadecylamine
(1.1 mg) were dissolved in chloroform. After the chloroform was removed by rotary evaporation, a thin lipid film remained in the flask. The film was hydrated with $1 \mathrm{~mL}$ of drug solution $(0.5 \mathrm{mg} / \mathrm{mL}$ for OVA and $0.4 \mathrm{mg} / \mathrm{mL}$ for PD) for $0.5 \mathrm{~h}$, and the particle size of OVA-PD-Lipos was reduced and made uniform by filtration through a series of polycarbonate membranes using a mini-extruder (Avestin Inc. Ottawa, Canada). Membrane pore sizes (in sequence) were 400, 200, and $100 \mathrm{~nm}$.

The mean particle size and zeta potential of OVA-PDLipos were measured by photo-correlation spectroscopy using a Nano ZS90 Zetasizer (Malvern Instruments Ltd, Worcestershire, UK). The entrapment efficiency of OVA in OVA-PD-Lipos was evaluated by filtering the OVA-PDLipos suspension through an ultracentrifugation tube (NMWL, 100 kDa, Merck KGaA, Darmstadt, Germany) at 4,500 rpm for $20 \mathrm{~min}$, and measuring the OVA level in the filtrate. OVA entrapment efficiency was calculated as follows:

$$
\text { Entrapment efficiency }(\mathrm{EE})=\frac{\left(\mathrm{W}_{\mathrm{t}}-\mathrm{W}_{\mathrm{f}}\right)}{\mathrm{W}_{\mathrm{t}}} \times 100 \%
$$

where $\mathrm{W}_{\mathrm{t}}$ is the total amount of OVA added and $\mathrm{W}_{\mathrm{f}}$ is the amount of free OVA.

The entrapment efficiency of PD in OVA-PD-Lipos was measured using the mini-column centrifugation method as described previously. ${ }^{13}$ OVA-PD-Lipos were separated from free PD by filtration through a Sephadex G-50-filling mini-column under centrifugation. The PD level in OVAPD-Lipos was determined using high-performance liquid chromatography, and entrapment efficiency was calculated accordingly.

\section{Hemolytic activity of OVA-PD-Lipos}

Rabbit RBCs were used to test whether incorporation of PD into liposomes reduces the hemolytic activity of OVAPD-Lipos. Rabbit blood was collected in a heparinized tube and fibers were removed by stirring the blood gently with a medical cotton stick. The RBCs were separated by centrifugation at 1,000 ref for $10 \mathrm{~min}$, then washed and centrifuged in normal saline four to five times. PD solution or OVA-PDLipos suspension $(40 \mu \mathrm{g} / \mathrm{mL})$ was incubated in three replicates with an equal volume of $1 \%$ RBC suspension for $30 \mathrm{~min}$ at $37^{\circ} \mathrm{C}$. Following incubation, supernatant from each sample was collected after centrifugation at 1,000 rcf for $10 \mathrm{~min}$, and absorbance was measured at $415 \mathrm{~nm}$. A suspension of empty liposomes served as measurement reference (blank), 
and normal saline and distilled water served as negative and positive controls, respectively.

\section{Toxicity of OVA-PD-Lipos}

Cultured mouse BMDCs were used to evaluate whether incorporation of $\mathrm{PD}$ into liposomes reduces the toxicity of OVA-PD-Lipos. Bone marrow cells were withdrawn from the femurs and tibias of male C57BL/ 6 mice provided by the Experimental Animal Center, Shanghai University of Traditional Chinese Medicine, as reported previously. ${ }^{14}$ Then, the cells were depleted of RBCs with RBC lysis buffer solution. BMDCs were prepared and maintained in RPMI 1640 medium containing $10 \%$ fetal bovine serum and $1 \%$ penicillin/streptomycin supplemented with recombinant murine GM-CSF (20 ng/mL) and $\beta$-mercaptoethanol $(50 \mathrm{nM})$. On days 3 and 5 , half of the medium was replaced with fresh RPMI 1640 containing $10 \%$ fetal bovine serum and $1 \%$ penicillin/streptomycin supplemented with recombinant murine GM-CSF (40 ng/mL) and $50 \mathrm{nM}$ or $50 \mu \mathrm{M} \beta$-ME. On day 7 , BMDCs were harvested and seeded into 96 -well plates at $1 \times 10^{5}$ cells per well, cultured for $24 \mathrm{~h}$, then incubated with OVA-PDLipos (1-50 $\mu \mathrm{g} / \mathrm{mL}, 6$ wells per level) for another $24 \mathrm{~h}$, with PD solution serving as a control. After the incubation, BMDC viability was measured using the MTT assay. ${ }^{15}$

\section{Uptake of OVA-PD-Lipos by BMDCs}

To evaluate whether the uptake of OVA is facilitated when loaded into liposomes, OVA was labeled with fluorescein isothiocyanate (FITC), and the uptake behavior of FITCOVA-PD-Lipos was investigated using mouse BMDCs.

FITC-OVA was prepared as described previously. ${ }^{16}$ Briefly, $10 \mathrm{mg}$ FITC and $50 \mathrm{mg}$ OVA were dissolved in $5 \mathrm{~mL}$ carbonate buffer solution (220 mM, pH 9.6), and kept under darkness with magnetic stirring at $4^{\circ} \mathrm{C}$ for $18 \mathrm{~h}$. Free FITC was removed by filtering the FITC-OVA solution through an ultracentrifugation tube (NMWL, 10 kDa, Merck KGaA) at 2,200 rcf for $20 \mathrm{~min}$.

Mouse BMDCs were seeded into a 6-well plate at $2 \times 10^{6}$ cells per well, and maintained in RPMI 1640 medium containing $10 \%$ fetal bovine serum and $1 \%$ penicillin/ streptomycin supplemented with recombinant murine GMCSF $(20 \mathrm{ng} / \mathrm{mL})$ and $\beta$-mercaptoethanol $(50 \mu \mathrm{M})$ for $24 \mathrm{~h}$. FITC-OVA or FITC-OVA-PD-Lipos $(100 \mu \mathrm{g} / \mathrm{mL})$ were added and incubated with the BMDCs for $4 \mathrm{~h}$. After incubation, BMDCs were separated, washed, and resuspended. The fluorescence of the suspensions was measured using a BD FACSCalibur flow cytometer (Becton, Dickinson and Company, Franklin Lakes, NJ, USA).

\section{Fabrication of OVA-PD-Lipos-MNs}

Hyaluronic acid microneedle arrays containing $200(10 \times 20)$ microneedles were used as master molds for fabricating OVA-PD-Lipos-MNs. Microneedle base diameter was $300 \mu \mathrm{m}$ and height was $356 \mu \mathrm{m}$. Polydimethylsiloxane (PDMS) was poured over the master mold and heated at $80^{\circ} \mathrm{C}$ for $3 \mathrm{~h}$. After the PDMS had cured thoroughly, the master mold was peeled off carefully, and the inverse microneedle mold (PDMS mold) was obtained.

OVA-PD-Lipos-MNs were fabricated as described previously with minor modifications. ${ }^{17}$ OVA-PD-Lipos were lyophilized and reconstituted in distilled water. The concentration of both OVA and PD was $2.5 \mathrm{mg} / \mathrm{mL}$. PVP-K17 was dissolved in OVA-PD-Lipos suspension (1:2, w/w), and PVP-K30 was dissolved in distilled water $(1: 1, w / w)$. These solutions served as the microneedle matrix solution and the base substrate solution, respectively. The microneedle matrix solution was added onto the surface of the PDMS mold, forced into the cavities using centrifugation, and then dried in a sealed desiccator after pipetting residual solution from the surface of the PDMS mold. The base substrate solution was added on the surface of the PDMS mold, air bubbles were removed under vacuum, and the preparation was dried thoroughly. Then, the OVA-PD-Lipos-MNs were peeled off from the PDMS mold.

\section{Physical stability of OVA-PD-Lipos in OVA-PD-Lipos-MNs}

The physical stability of OVA-PD-Lipos in OVA-PDLipos-MNs was evaluated as described previously. ${ }^{17} \mathrm{OVA}-$ PD-Lipos-MNs were dissolved in distilled water to release OVA-PD-Lipos. The morphology of the OVA-PD-Lipos after release was observed using a JEM-1400 transmission electron microscope (JEOL Ltd., Tokyo, Japan), and compared with empty liposomes or a suspension of OVA-PDLipos that had not been associated with MNs.

\section{Chemical stability of OVA in OVA-PD-Lipos-MNs}

The chemical stability of OVA in OVA-PD-Lipos-MNs was investigated using sodium dodecyl sulfate polyacrylamide gel electrophoresis (SDS-PAGE), with free OVA and OVA-PDLipos as controls. OVA was freed from OVA-PD-Lipos or OVA-PD-Lipos-MNs (after dissolving in distilled water) by the addition of $10 \%$ triton (w/w). SDS-PAGE was conducted using a 5\% stacking gel and 10\% separation gel. Samples were heated to $100^{\circ} \mathrm{C}$, maintained at this temperature for $10 \mathrm{~min}$, loaded onto the vertical slab gel, and subjected to electrophoresis at 
$60 \mathrm{mV}$ for $30 \mathrm{~min}$ and then at $80 \mathrm{mV}$. After electrophoresis, gels were fixed and stained with Coomassie brilliant blue.

\section{Insertion of OVA-PD-Lipos-MNs into skin}

In vitro and in vivo experiments were conducted to evaluate whether OVA-PD-Lipos-MNs have sufficient mechanical strength to penetrate skin, dissolve rapidly, and release the subunit vaccine. To test skin penetration, OVA-PDLipos-MNs were inserted into mouse skin in vitro and held for $2 \mathrm{~min}$. The treated mouse skin was stained with $0.4 \%$ trypan blue to observe the penetration site directly, or embedded into paraffin, cut into vertical slices, stained with hematoxylin-eosin, and observed using microscope. To test dissolving properties, OVA-PD-Lipos-MNs were inserted into mouse skin in vivo, held for 10,30 , or $60 \mathrm{~s}$, and then the insertion site was imaged using a scanning electron microscope (JEM-1230, JEOL Ltd.). Finally, to test delivery efficiency, FITC-OVA-PD-Lipos was prepared as described and loaded into dissolving microneedle arrays. The arrays were inserted into nude mouse skin in vivo. The nude mice were provided by the Experimental Animal Center, Shanghai University of Traditional Chinese Medicine. As a control, medical gauze soaked with FITC-OVA-PD-Lipos suspension was applied to the skin. After $30 \mathrm{~min}$, fluorescence intensity of the skin sample was measured using a fluorescence imaging system (IVIS Lumina XR, Caliper Life Sciences, Inc., Waltham, MA, USA).

\section{Immunization of mice with OVA-PD- Lipos-MNs}

The immunization efficiency of OVA-PD-Lipos-MNs was evaluated in Institute of Cancer Research (ICR) mice. Female mice (18 \pm 2 g, 5 weeks old) were obtained from the Experimental Animal Center, Shanghai University of Traditional Chinese Medicine, and divided randomly into 6 groups: OVA, OVA + PD, OVA-PD-Lipos, OVA-MNs, OVA-PDMNs, and OVA-PD-Lipos-MNs ( $n=6$ per group). Animals in each group were immunized first with $25 \mu \mathrm{g}$ OVA and/or PD, and boosted with $25 \mu \mathrm{g}$ OVA and/or PD 2 weeks later. Animals in the OVA, OVA + PD, and OVA-PD-Lipos groups were injected subcutaneously with OVA solution, OVA and PD solution, or OVA-PD-Lipos, respectively. Animals in the OVA-MNs, OVA-PD-MNs, and OVA-PD-Lipos-MNs groups were administered with OVA-MNs (free OVA-loaded dissolving microneedles), OVA-PD-MNs (free OVA- and PDloaded dissolving microneedles), or OVA-PD-Lipos-MNs, respectively. Two weeks after the second immunization, blood samples were drawn from mice in each group, and centrifuged at 3,000 rpm for $20 \mathrm{~min}$ to obtain serum. OVA-specific $\mathrm{IgG}$, IgG1, and IgG2b levels in the serum samples were measured using ELISA kits (Shanghai Xin Yu Biotech Co., Ltd., Shanghai, China), following the manufacturer's instructions.

\section{Evaluation of skin irritation caused by OVA-PD-Lipos-MNs}

The potential for OVA-PD-Lipos-MNs to irritate normal skin after multiple-dose administration was investigated in accordance with OECD Guideline 404, "Acute Dermal Irritation/Corrosion". ${ }^{18}$ New Zealand rabbits $(2.0-2.5 \mathrm{~kg})$ were divided randomly into four groups (blank control, blank MNs, OVA-PD-MNs, and OVA-PD-Lipos-MNs; $\mathrm{n}=4$ animals per group). A small region on the left flank of each rabbit $(\sim 3 \times 3 \mathrm{~cm})$ was shaved with an electric clipper. After the rabbits had recovered for $24 \mathrm{~h}$, they were inspected visually for skin damage. Rabbits without skin damage were enrolled in the experiments. Rabbits in the blank MNs, OVAPD-MNs, and OVA-PD-Lipos-MNs groups were treated with blank MNs, OVA-PD-MNs (free OVA- and PD-loaded microneedles), or OVA-PD-Lipos-MNs, respectively, once daily for 3 consecutive days. The microneedles were inserted into shaved rabbit skin, held for $3 \mathrm{~min}$, fixed with medical tape for $4 \mathrm{~h}$ and then removed. After removal, the treated skin was cleaned with warm distilled water. The treated area was inspected visually for skin irritation and images were recorded before each treatment, $1 \mathrm{~h}$ after microneedle removal following the first and second treatments, and 1 , 24, 48 and $72 \mathrm{~h}$ after removal of the microneedles following the final treatment. Rabbits in the blank control group were observed in parallel with the other groups.

The Institutional Animal Ethical Committee of the Shanghai University of Traditional Chinese Medicine approved the experimental procedures and all procedures followed the guidelines of the Institutional Animal Ethical Committee of the Shanghai University of Traditional Chinese Medicine.

\section{Statistics}

Data are expressed as the mean $\pm \mathrm{SD}$. One-way analysis of variance was used to determine significance among the treatment groups, after which least significant difference$t$-test were used for comparison between individual groups. A value of $P<0.05$ was considered to be significant.

\section{Results and discussion Characterization of OVA-PD-Lipos}

The mean particle size of OVA-PD-Lipos was $135.8 \mathrm{~nm}$. The liposomes were relatively uniform in size, as indicated 
by their small polydispersity index (0.137). The Zeta potential of OVA-PD-Lipos was $45.7 \mathrm{mV}$, which might be attributed to the addition of octadecylamine in the formulation of OVA-PD-Lipos. The entrapment efficiencies of OVA and PD in OVA-PD-Lipos were $58.98 \% \pm 4.01 \%$ and $46.19 \% \pm 2.65 \%$, respectively.

\section{Hemolytic activity of OVA-PD-Lipos}

Hemolysis is the primary toxic effect of PD and other saponin adjuvants..$^{13}$ Using rabbit RBCs, the hemolytic activities of OVA-PD-Lipos, PD solution, blank liposomes, and normal saline were compared. As shown in Figure 1, PD solution caused severe hemolytic activity, as indicated by its high hemolysis percentage (nearly $70 \%$ ), whereas OVA-PD-Lipos exhibited far lower hemolytic activity $(<7 \%)$, similar to blank liposomes and normal saline. This result demonstrates that the hemolytic effects of PD can be reduced dramatically when PD is incorporated into liposomes, consistent with previous reports. ${ }^{13}$

\section{Toxicity of OVA-PD-Lipos to BMDCs}

Because DCs play a vital role in the elicitation of the immune response by vaccines, it is important to demonstrate that their function is not compromised by OVA-PD-Lipos. OVA-PD-Lipos toxicity was tested using cultured mouse BMDCs, and compared to the toxicity exhibited by PD solution. As shown in Figure 2, free PD exhibited obvious toxicity to BMDCs at concentrations over $5 \mu \mathrm{g} / \mathrm{mL}$, and BMDC viability was $<10 \%$ when the concentration of free PD reached $20 \mu \mathrm{g} / \mathrm{mL}$. In contrast, OVA-PD-Lipos exhibited minimal toxicity to BMDCs, and BMDC viability was never below $70 \%$ across the range of $\mathrm{PD}$ concentrations tested $(1-50 \mu \mathrm{g} / \mathrm{mL})$. These data demonstrate that PD

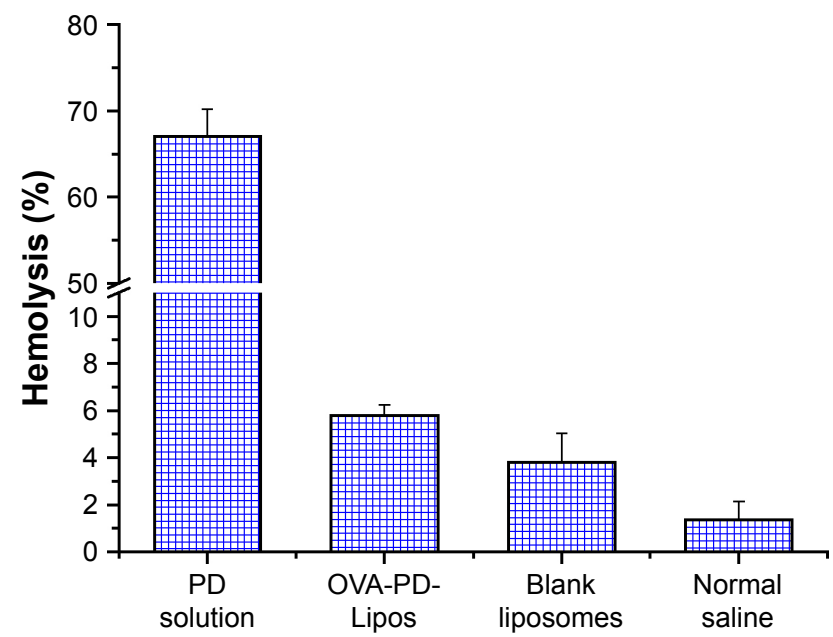

Figure I Hemolytic activity of OVA-PD-Lipos in rabbit RBCs $(n=3)$. Abbreviations: OVA, ovalbumin; PD, Platycodin D; RBCs, red blood cells.

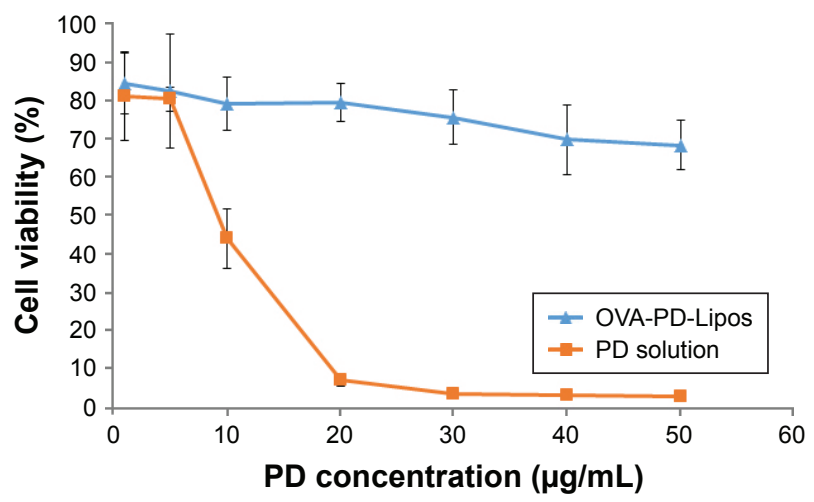

Figure 2 Toxicity of OVA-PD-Lipos to cultured mouse BMDCs $(n=6)$. Abbreviations: BMDCs, bone marrow dendritic cells; OVA, ovalbumin; PD, Platycodin D.

is dramatically reduced in toxicity when incorporated into liposomes. Interactions between saponin and cholesterol in the cell membrane, and the resulting membrane damage, may underlie saponin-induced hemolysis and other toxicities. ${ }^{19}$ The incorporation of PD into OVA-PD-Lipos may reduce interactions with cholesterol and account for the reduction in toxicity to mouse BMDCs as well as the reduction in hemolytic activity.

\section{Uptake of OVA-PD-Lipos by BMDCs}

The uptake and presentation of subunit vaccine by DCs are crucial steps in subunit vaccine mediated immunization. The uptake behavior of OVA-PD-Lipos was investigated in comparison with OVA solution, using cultured mouse BMDCs as a model. OVA uptake increased more than one order of magnitude when OVA was incorporated into liposomes (Figure 3). A possible explanation is that uptake through endocytosis is facilitated by nanoscale carriers

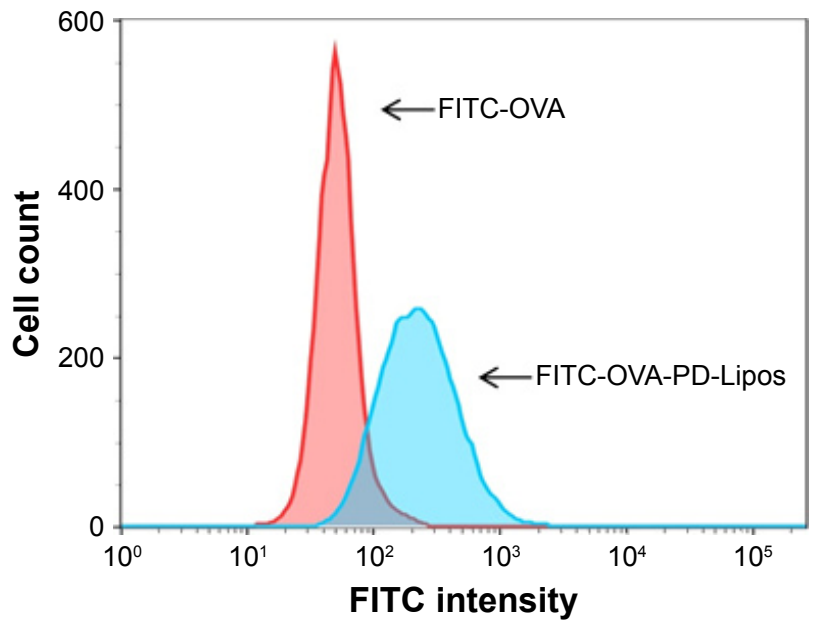

Figure 3 Uptake of FITC-OVA-PD-Lipos and FITC-OVA by cultured mouse BMDCs as determined by flow the units for the $y$-axes of this figure are cell count. Abbreviations: BMDCs, bone marrow dendritic cells; FITC, fluorescein isothiocyanate; PD, Platycodin D; OVA, ovalbumin. 

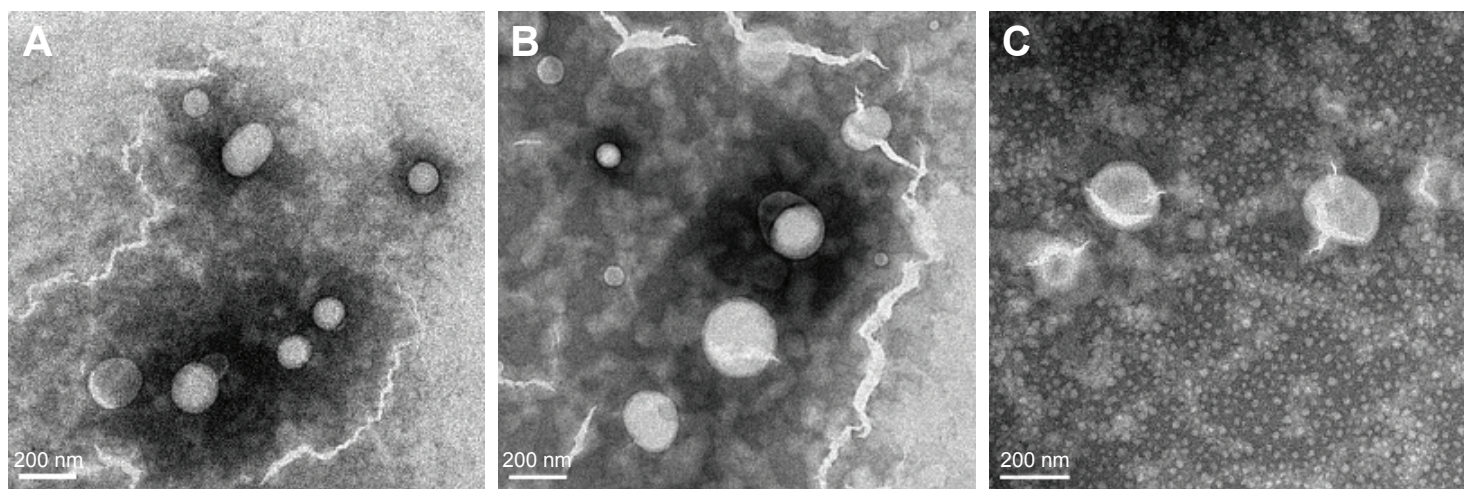

Figure 4 Transmission electron micrographs of (A) empty liposomes, (B) OVA-PD-Lipos, and (C) OVA-PD-Lipos-MNs. Note: TEM was performed after OVA-PD-Lipos-MNs were dissolved in distilled water.

Abbreviations: OVA, ovalbumin; PD, Platycodin D; MNs, microneedle array.

loaded with subunit vaccines, as reported previously. ${ }^{20}$ Recently, it has been reported that the targeting efficiency of nanoparticles to DCs might be improved by surface modification of ligands to receptors overexpressed on DC's surface. ${ }^{21}$ To further improve the DC targeting efficiency of subunit vaccine delivery system, surfacially modified nanoparticles might be incorporated into the dissolving microneedle array.

\section{Physical stability of OVA-PD-Lipos in OVA-PD-Lipos-MNs}

The physical stability of OVA-PD-Lipos in OVA-PDLipos-MNs was investigated using transmission electron microscopy. Loaded microneedles were dissolved in distilled water, and their morphology was compared with suspensions of OVA-PD-Lipos and blank liposomes. OVA-PD-Lipos released from dissolving microneedles were smooth, spherical particles, relatively uniform in size, and nearly identical in appearance to OVA-PD-Lipos and blank liposomes (Figure 4). OVA-PD-Lipos in suspension and after release from dissolving microneedle arrays were slightly larger than empty liposomes, possibly due to the incorporation of OVA and PD. These results suggest that OVA-PD-Lipos in dissolving microneedle arrays exhibit good physical stability.

\section{Chemical stability of OVA in OVA-PD-Lipos-MNs}

The chemical stability of OVA in OVA-PD-Lipos-MNs was investigated using SDS-PAGE. As shown in Figure 5, only one band was observed in lanes containing OVA-PD-Lipos or OVA-PD-Lipos-MNs. The bands comigrate with the 44-kDa protein marker, which has a similar molecular weight as free OVA (lane B). This result indicates that OVA has good chemical stability when OVA-PD-Lipos are incorporated into dissolving microneedles.

\section{Insertion of OVA-PD-Lipos-MNs into skin}

The behavior of OVA-PD-Lipos-MNs inserted into skin was investigated in vitro and in vivo. As shown in Figure 6, an array of needle holes is observed after insertion of OVA-PDLipos-MNs into mouse skin in vitro. Obvious needle holes are also visible in a vertical slice through the treated skin. These results demonstrate that OVA-PD-Lipos-MNs insert reliably into skin. OVA delivery by the microneedles was tested in vivo using FITC-OVA. Nude mice treated with

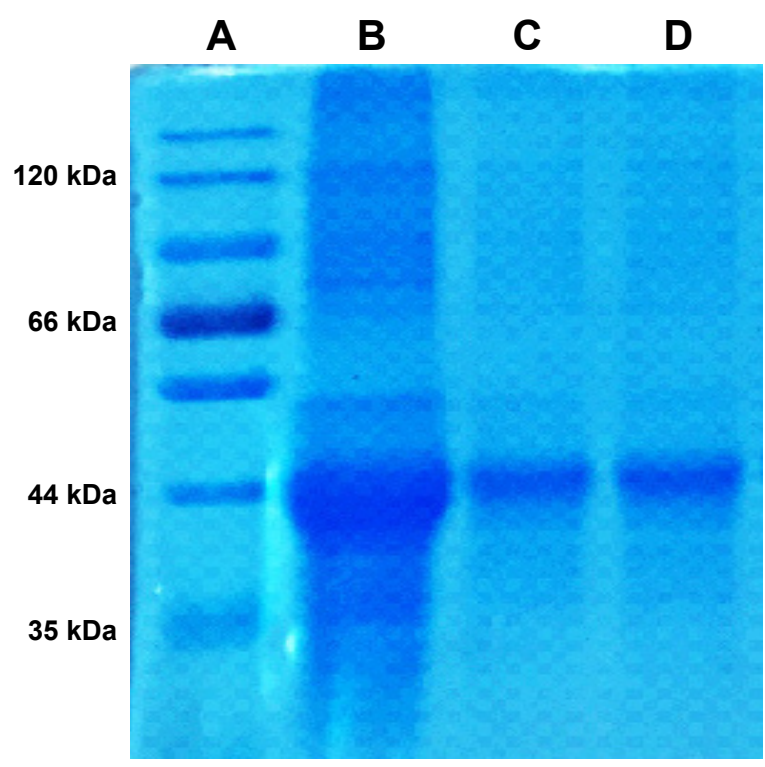

Figure 5 The integrity of OVA in OVA-PD-Lipos-MNs analyzed by SDS-PAGE. Notes: Lane (A) protein molecular mass markers; Lane (B) OVA standard; Lane (C) OVA-PD-Lipos; Lane (D) OVA-PD-Lipos-MNs.

Abbreviations: OVA, ovalbumin; PD, Platycodin D; SDS-PAGE, sodium dodecyl sulfate polyacrylamide gel electrophoresis; MNs, microneedle array. 

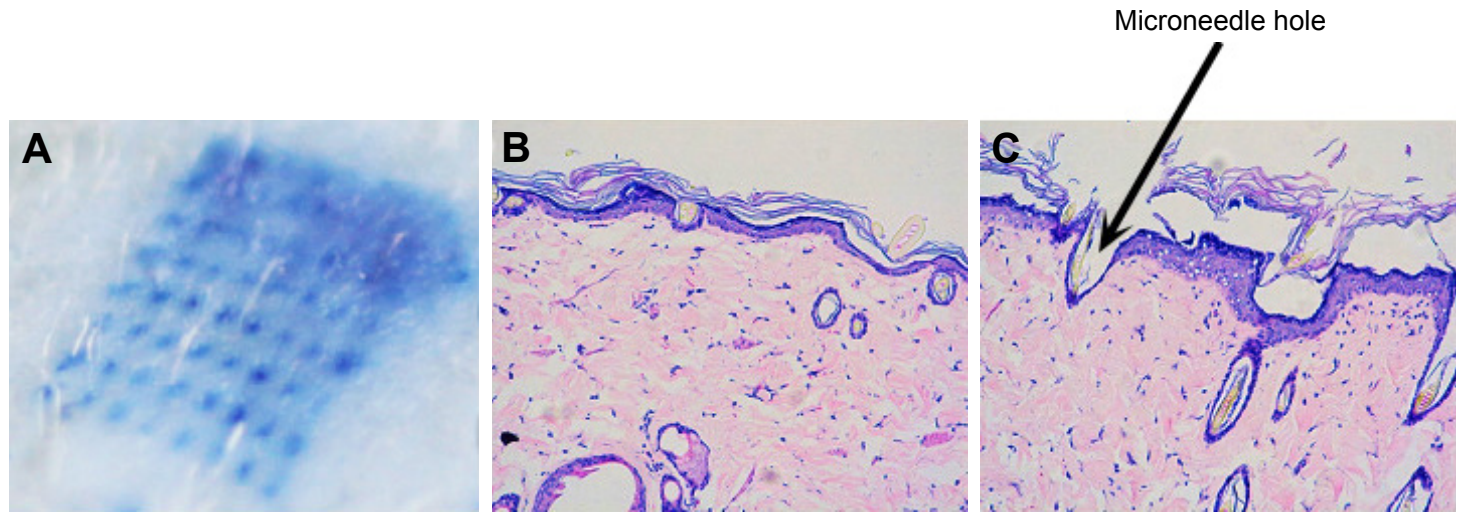

Figure 6 Evaluation of OVA-PD-Lipos-MNs inserted into mouse skin in vitro.

Notes: (A) Array of needle holes in mouse skin stained with trypan blue after insertion. (B and C) Vertical slices of mouse skin before and after insertion of OVA-PDLipos-MNs (stained using H\&E).

Abbreviations: H\&E, hematoxylin-eosin; OVA, ovalbumin; PD, Platycodin D; MNs, microneedle array.

FITC-OVA-PD-Lipos-MNs exhibited intense fluorescence, showing that the dissolving microneedle arrays effectively deliver OVA into skin (Figure 7). In contrast, no fluorescence is observed if the skin is treated using FITC-OVA-PD-Lipos, demonstrating that OVA penetrates minimally into intact skin through passive diffusion, even if incorporated into liposomes.
The exclusion is probably due to the high molecular weight of FITC-OVA-PD-Lipos. As shown in Figure 8, OVA-PDLipos-MNs dissolve within 1 min after insertion into mouse skin in vivo. Together, these results demonstrate that the dissolving microneedle array system can effectively deliver, and rapidly release, subunit vaccines into skin.

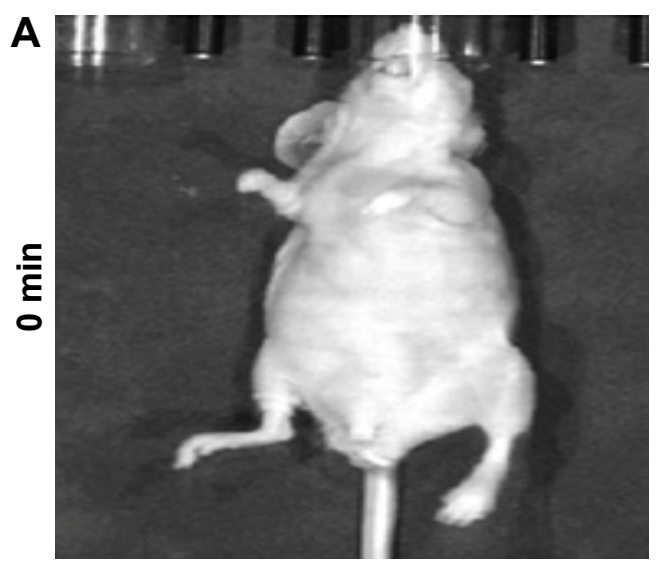

B
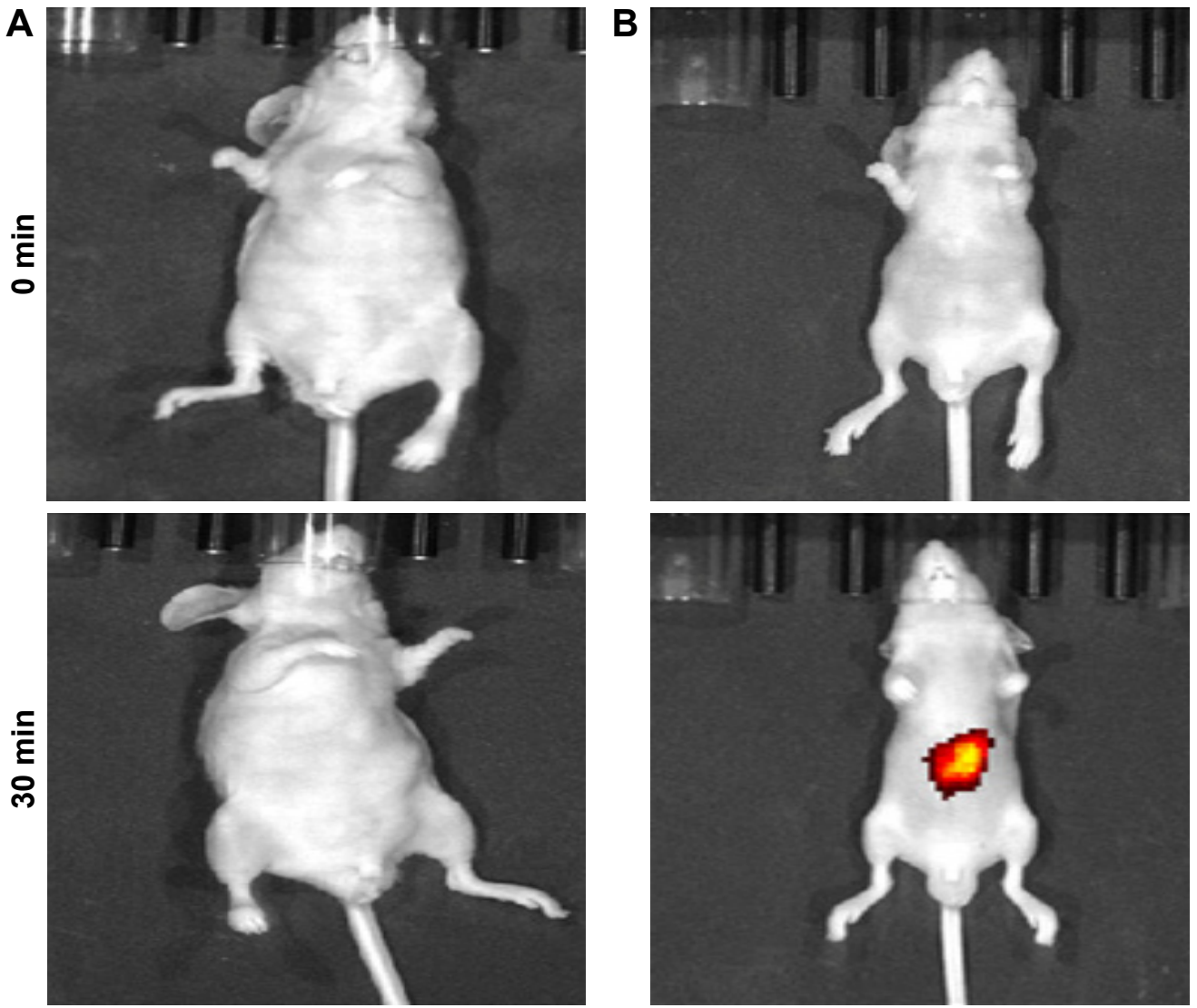

Figure 7 Images of nude mice after topical application of (A) FITC-OVA-PD-Lipos and (B) FITC-OVA-PD-Lipos-MNs. The colored area represents fluorescent intensity. Abbreviations: FITC, fluorescein isothiocyanate; OVA, ovalbumin; PD, Platycodin D; MNs, microneedle array. 
$0 \mathrm{~s}$

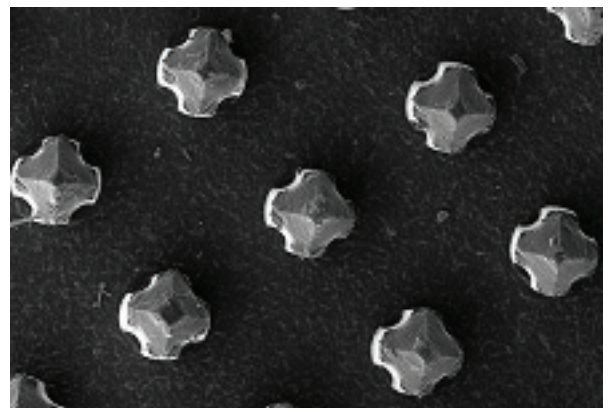

$30 \mathrm{~s}$

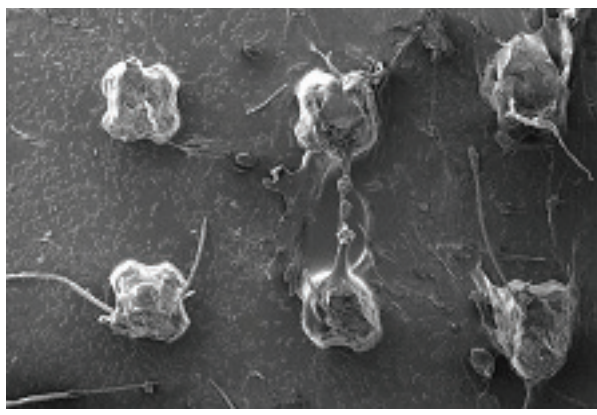

$10 \mathrm{~s}$

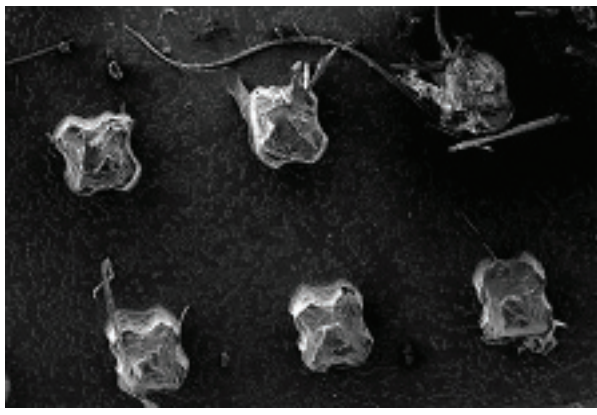

$60 \mathrm{~s}$

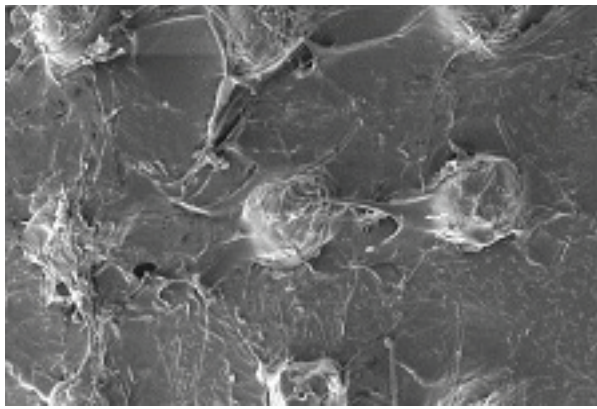

Figure 8 Dissolution of OVA-PD-Lipos-MNs after insertion into mouse skin in vivo. Abbreviations: OVA, ovalbumin; PD, Platycodin D; MNs, microneedle array.
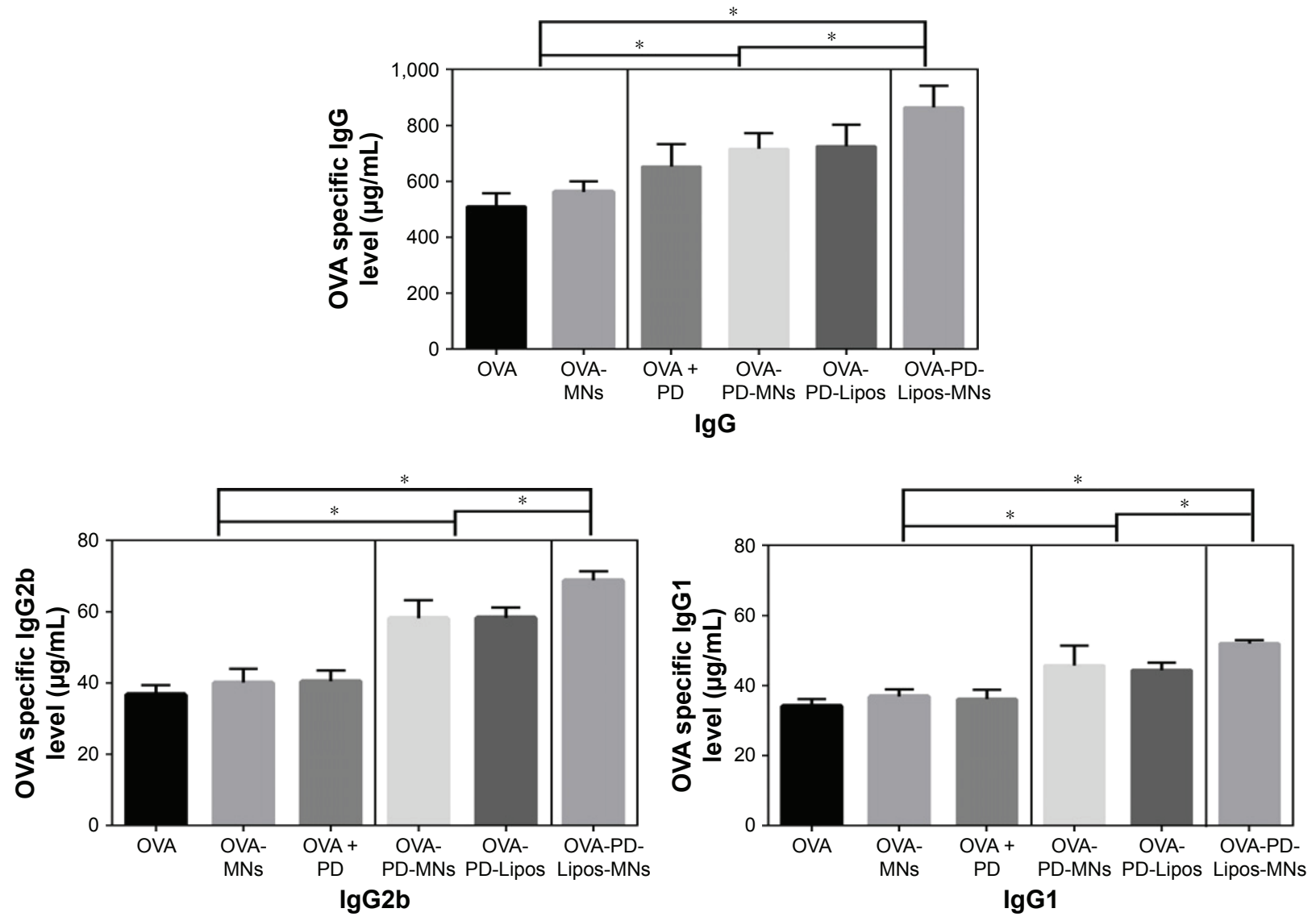

Figure 9 OVA-specific lgG, $\operatorname{lgG} I$ and $\lg G 2 b$ levels in ICR mice immunized with OVA-PD-Lipos-MNs $(\mathrm{n}=6)$.

Notes: OVA, OVA + PD, and OVA-PD-Lipos represent OVA solution, OVA and PD solution, OVA and PD loaded liposomes, respectively; OVA-MNs, OVA-PD-MNs, and OVA-PD-Lipos-MNs represent free OVA, free OVA + PD, and OVA-PD-Lipos, loaded into dissolving microneedle array, separately; no statistical difference was found among groups in the same box; $* P<0.05$.

Abbreviations: OVA, ovalbumin; PD, Platycodin D; MNs, microneedle array. 


\section{Immunization of mice using OVA-PD- Lipos-MNs}

The immunization efficiency of OVA-PD-Lipos-MNs was compared in ICR mice with five preparations: free OVA, free OVA loaded into dissolving microneedles, OVA- and PDloaded into dissolving microneedles, free OVA plus $\mathrm{PD}$, and OVA-PD-Lipos (OVA, OVA-MNs, OVA-PD-MNs, OVA + $\mathrm{PD}$, and OVA-PD-Lipos, respectively). After immunization, OVA specific IgG, IgG1, and IgG2b levels in mouse serum were measured (Figure 9).

The lowest mean OVA-specific IgG levels were seen in mice immunized with OVA solution alone or with OVA-MNs. These were statistically indistinguishable, but were significantly different $(P<0.05)$ from the higher OVA-specific IgG levels observed in mice immunized with PD + OVA, OVA-PD-MNs, or OVA-PD-Lipos. The difference demonstrates that immunization efficiency is improved when OVA is combined with adjuvant. The highest OVA-specific IgG level, exhibited by mice immunized with OVA-PD-Lipos-MNs, was significantly different from all the others $(P<0.05)$. Immunization efficiency is clearly enhanced when OVA is combined with adjuvant and an effective delivery system. This is consistent with results presented earlier showing that loading OVA-PD-Lipos into dissolving microneedle arrays enhances physical targeting efficiency (Figure 7), and with our results showing that incorporating OVA and PD into liposomes enhances uptake of OVA-PD-Lipos by BMDCs (Figure 3), possibly via endocytosis. It is, therefore, likely that the high OVAspecific IgG level exhibited by mice immunized with OVAPD-Lipos-MNs is the result of increased uptake of OVA by DCs, due to the microneedle delivery system as well as the positive immunogenic effect of $\mathrm{PD}$ adjuvant.

The results obtained for IgG1 or IgG2b levels were similar to those obtained for IgG, except that IgG1 or IgG2b levels in mice immunized with OVA versus OVA + PD were statistically indistinguishable. OVA's immunization efficiency is only slightly enhanced when it is simply combined with adjuvant. Alternatively, the IgG1 and IgG2b subclasses of IgG may be less responsive to adjuvant than IgG. Compared with mice immunized with OVA alone (OVA solution or OVA-MNs), higher levels of IgG1 and IgG2b were observed in all mice when OVA was combined with PD, except for mice immunized with OVA + PD. This suggests that PD not only increases OVA's immunization efficiency, but also elicits a balanced Th1 and Th2 type humoral immunity response, consistent with suggestions that PD can be used as an adjuvant in both prophylactic and therapeutic vaccines. ${ }^{3}$

\section{Evaluation of skin irritation caused by OVA-PD-Lipos-MNs}

Because a goal of this study is to evaluate PD-Lipos-MNs as a vehicle for the intradermal delivery of vaccines, the ability of OVA-PD-Lipos-MNs to provoke skin irritation was investigated using rabbits. Animals were treated with OVA-PD-Lipos-MNs and controls once daily for three

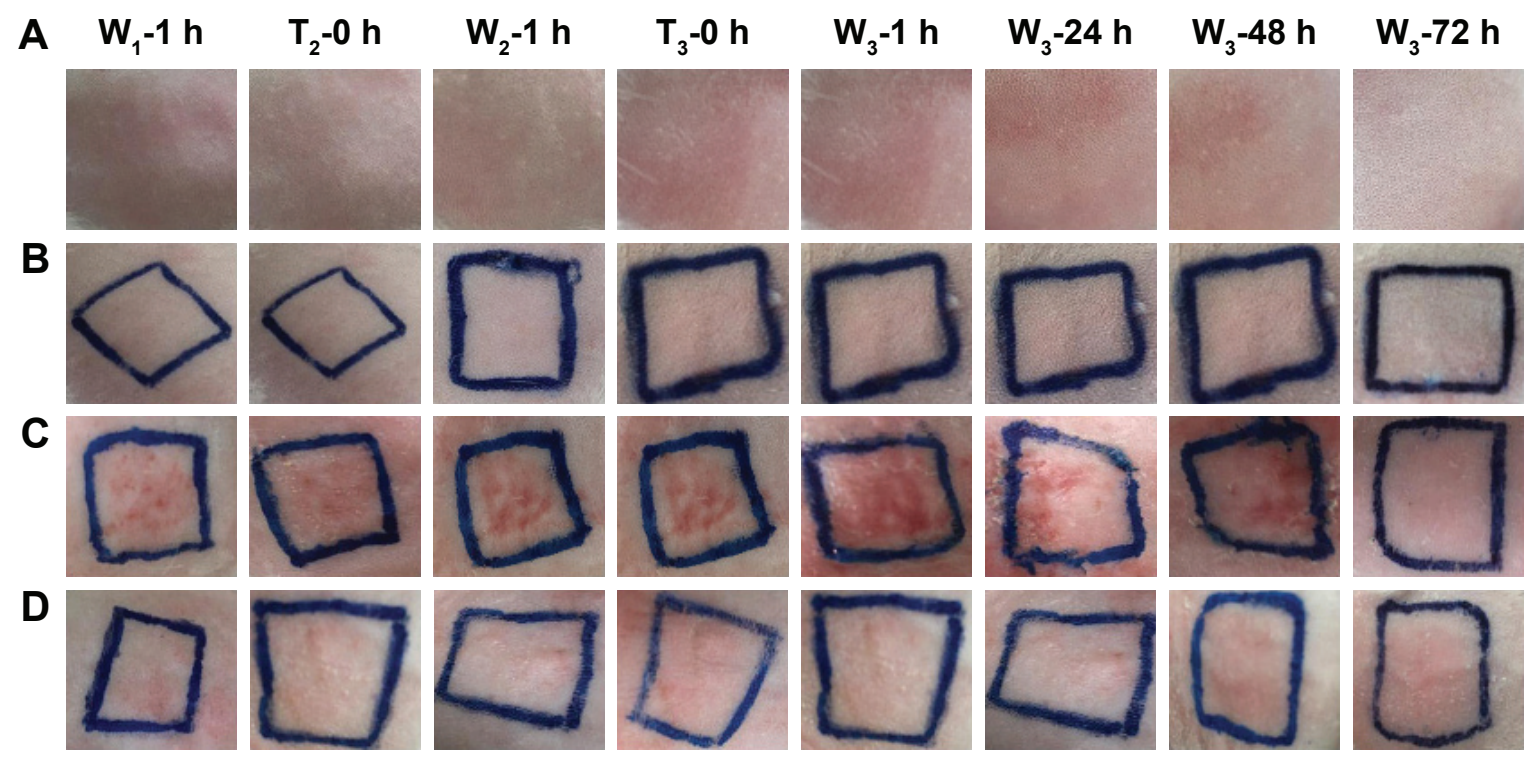

Figure 10 Response of intact rabbit skin after multiple-dose delivery of OVA-PD-Lipos-MNs.

Notes: Rows A-D represent blank control, blank dissolving microneedles, free OVA and PD in dissolving microneedles, and OVA-PD-Lipos-MNs, respectively. Column W, I $\mathrm{h}$ and $W_{2}-I$ h represent I $h$ after the washing out of the first and second dose; Column $W_{3}-I ~ h, W_{3}-24 h, W_{3}-48 h$, and $W_{3}-72 h$ represent I, 24,48 , and $72 \mathrm{~h}$ after the washing out of the third dose, respectively. Column $\mathrm{T}_{2}-0 \mathrm{~h}$ and $\mathrm{T}_{3}-0 \mathrm{~h}$ represent immediately before the application of the second and third dose.

Abbreviations: OVA, ovalbumin; PD, Platycodin D; MNs, microneedle array. 
consecutive days and then visually monitored (Figure 10). Microneedles loaded with PD + OVA produced the most serious irritation response, which disappeared $72 \mathrm{~h}$ after the last treatment. In contrast, no skin irritation occurred in untreated rabbits (blank control group), in rabbits treated with blank dissolving microneedle arrays, or in those treated with OVA-PD-Lipos-MNs. These results demonstrate that PD toxicity is reduced dramatically when incorporated into liposomes and loaded into dissolving microneedle arrays, and are consistent with the hemolytic activity and cell toxicity experiments described earlier.

\section{Conclusion}

The immune response of ICR mice to the model subunit vaccine OVA was significantly enhanced when OVA and its adjuvant were incorporated together into a dissolving microneedle delivery system. PD, a novel saponin adjuvant, elicited a balanced Th1 and Th2 type humoral immune response when combined with OVA, and may serve as potent adjuvant for both prophylactic and therapeutic vaccines. The dissolving microneedle array based system is a promising delivery vehicle for a subunit vaccine and its adjuvant.

\section{Acknowledgment}

This work was supported by the Program from Shanghai University of Traditional Chinese Medicine (No 2015YSN09).

\section{Disclosure}

The authors report no conflicts of interest in this work.

\section{References}

1. Drane D, Gittleson C, Boyle J, Maraskovsky E. ISCOMATRIX adjuvant for prophylactic and therapeutic vaccines. Expert Rev Vaccines. 2007;6(5):761-772.

2. Song X, Hu S. Adjuvant activities of saponins from traditional Chinese medicinal herbs. Vaccine. 2009;27(36):4883-4890.

3. Xie Y, Sun HX, Li D. Platycodin D is a potent adjuvant of specific cellular and humoral immune responses against recombinant hepatitis B antigen. Vaccine. 2009;27(5):757-764.

4. Silva JM, Zupancic E, Vandermeulen G, et al. In vivo delivery of peptides and Toll-like receptor ligands by mannose-functionalized polymeric nanoparticles induces prophylactic and therapeutic anti-tumor immune responses in a melanoma model. $J$ Control Release. 2015;198:91-103.

5. Kastenmüller W, Kastenmüller K, Kurts C, et al. Dendritic cell-targeted vaccines - hope or hype? Nat Rev Immunol. 2014;14(10):705-711.
6. Marcel B, Teunissen M. Current Topics in Microbiology and Immunology Vol 351: Intradermal Immunization. Switzerland: Springer. 2011.

7. Madhusudana SN, Mani RS. Intradermal vaccination for rabies prophylaxis: conceptualization, evolution, present status and future. Expert Rev Vaccines. 2014;13(5):641-655.

8. Arya JM, Dewitt K, Scott-Garrard M, Chiang YW, Prausnitz MR. Rabies vaccination in dogs using a dissolving microneedle patch. J Control Release. 2016;239:19-26.

9. Esser ES, Romanyuk A, Vassilieva EV, et al. Tetanus vaccination with a dissolving microneedle patch confers protective immune responses in pregnancy. J Control Release. 2016;236:47-56.

10. Zaric M, Lyubomska O, Touzelet $\mathrm{O}$, et al. Skin dendritic cell targeting via microneedle arrays laden with antigen-encapsulated poly-D,Llactide-co-glycolide nanoparticles induces efficient antitumor and antiviral immune responses. ACS Nano. 2013;7(3):2042-2055.

11. Sullivan SP, Koutsonanos DG, Del Pilar Martin M, et al. Dissolving polymer microneedle patches for influenza vaccination. Nat Med. 2010;16(8):915-920.

12. Xiong D, Liu Z, Bian T, et al. GX1-mediated anionic liposomes carrying adenoviral vectors for enhanced inhibition of gastric cancer vascular endothelial cells. Int J Pharm. 2015;496(2):699-708.

13. Xiong Y, Guo D, Zheng XL, Sun P, Xu LY, Chen JM. [Preliminary study on sea cucumber saponin-nobiliside A liposome and its hemolytic activity]. Yао Хие Bao. 2008;43(2):214-220. Chinese.

14. Winzler C, Rovere P, Rescigno M, et al. Maturation stages of mouse dendritic cells in growth factor-dependent long-term cultures. $J$ Exp Med. 1997;185(2):317-328.

15. Scherliess R. The MTT assay as tool to evaluate and compare excipient toxicity in vitro on respiratory epithelial cells. Int J Pharm. 2011; 411(1-2):98-105.

16. Demana PH, Davies NM, Hook S, Rades T. Quil A-lipid powder formulations releasing ISCOMs and related colloidal structures upon hydration. J Control Release. 2005;103(1):45-59.

17. Guo L, Chen J, Qiu Y, et al. Enhanced transcutaneous immunization via dissolving microneedle array loaded with liposome encapsulated antigen and adjuvant. Int $J$ Pharm. 2013;447(1-2):22-30.

18. Ema M, Matsuda A, Kobayashi N, Naya M, Nakanishi J. Evaluation of dermal and eye irritation and skin sensitization due to carbon nanotubes. Regul Toxicol Pharmacol. 2011;61(3):276-281.

19. Lorent JH, Quetin-Leclercq J, Mingeot-Leclercq MP. The amphiphilic nature of saponins and their effects on artificial and biological membranes and potential consequences for red blood and cancer cells. Org Biomol Chem. 2014;12(44):8803-8822.

20. Uto T, Akagi T, Toyama M, et al. Comparative activity of biodegradable nanoparticles with aluminum adjuvants: antigen uptake by dendritic cells and induction of immune response in mice. Immunol Lett. 2011;140(1-2):36-43.

21. Cruz LJ, Rosalia RA, Kleinovink JW, Rueda F, Lowik CW, Ossendorp F. Targeting nanoparticles to CD40, DEC-205 or CD11c molecules on dendritic cells for efficient CD8(+) T cell response: a comparative study. J Control Release. 2014;192:209-218.

\section{Publish your work in this journal}

The International Journal of Nanomedicine is an international, peerreviewed journal focusing on the application of nanotechnology in diagnostics, therapeutics, and drug delivery systems throughout the biomedical field. This journal is indexed on PubMed Central, MedLine, CAS, SciSearch $\AA$, Current Contents $₫ /$ Clinical Medicine,

\section{Dovepress}

Journal Citation Reports/Science Edition, EMBase, Scopus and the Elsevier Bibliographic databases. The manuscript management system is completely online and includes a very quick and fair peer-review system, which is all easy to use. Visit http://www.dovepress.com/ testimonials.php to read real quotes from published authors. 